STRUCTURAL BIOLOGY COMMUNICATIONS

ISSN 2053-230X

Received 27 January 2016

Accepted 10 March 2016

Edited by W. N. Hunter, University of Dundee, Scotland

Keywords: HINT; PKCI.

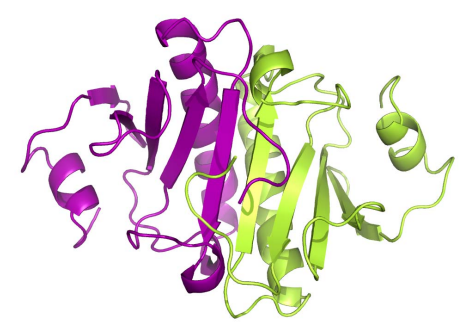

C 2016 International Union of Crystallography

\section{Errors in Crystal structure of HINT from Helicobacter pylori}

\author{
Kimberly M. Maize*
}

Medicinal Chemistry, University of Minnesota, 308 Harvard St SE, 8-101 Weaver-Densford Hall, Minneapolis, Minnesota 55455, USA. *Correspondence e-mail: maize001@umn.edu

Inaccuracies in the article, Crystal structure of HINT from Helicobacter pylori by Tarique et al. [(2016) Acta Cryst. F72, 42-48] are presented, and a brief history of HINT nomenclature is discussed.

The article, Crystal structure of HINT from Helicobacter pylori by K. F. Tarique et al. (2016) has come to my attention as containing several factual errors in the Introduction regarding the nature and annotation of HINT enzymes. As an author cited by this paper, I feel that it is necessary to provide corrections for the benefit of interested readers.

The HINT proteins are well established as performing hydrolysis on a wide variety of phosphoramidates and acyl-nucleotides (Bieganowski et al., 2002; Chou et al., 2007; Chou \& Wagner, 2007). Interest in these enzymes has renewed recently as HINT1 is responsible for the cellular activation of blockbuster antiviral phosphoramidate nucleotide prodrug sofosbuvir by cleaving the bond between the phosphorous and nitrogen (Murakami et al., 2010). Tarique et al. mischaracterize the catalysis as hydrolysis of the adenine nucleobase from the ribose and identify the substrate of the HINT proteins as phosphoramidites, an error in substrate oxidation state.

The structure of the protein now known as HINT1 (also hHint1) was first solved in 1996 at Columbia by the Hendrickson group. The bovine homolog of this protein had been identified biochemically as an inhibitor of protein kinase C (PKC) (McDonald \& Walsh, 1985), giving rise to the initial annotation of protein kinase $\mathrm{C}$ interacting protein 1 (PKCI-1) in three associated PDB structures $(1 \mathrm{kpa}, 1 \mathrm{kpb}$, and $1 \mathrm{kpc})$. However, even in this first paper, there was doubt about the veracity of the PKC activity, '... other investigators have characterized a protein similar to bovine PKCI-1 from other organisms and have not been able to demonstrate inhibitory effect on PKC activity... In addition, some doubt has been cast on the physiological relevance of PKC inhibition by bovine PKCI-1' (Lima et al., 1996). A year later, an enzymatic function of nucleotide diphosphate hydrolysis was demonstrated (associated structures 1av5, 1kpe, and 1kpf) (Lima et al., 1997).

That same year, the Petsko group at Brandeis also purified and crystallized rabbit HINT (associated structures 3rhn, 4rhn, 5rhn, 6rhn), saying, 'HINT is nearly identical to proteins that have been given the designation protein kinase $\mathrm{C}$ inhibitor-1 (PKCI-1). Bovine PKCI-1 was so named because it was present in brain cytosol fractions that inhibited a mixture of PKC isoforms (McDonald \& Walsh, 1985). It has not been possible to reproduce this inhibition with HINT from rabbit heart or with purified recombinant HINT.' (Brenner et al., 1997) It was this publication that gave HINT proteins their name.

In 1999, Brenner et al. devoted an entire section of a review article to the pervasive annotation of HINT as PKCI-1, insisting that 'Hint is not a PKC inhibitor' (Brenner et al., 1999). They noted that this enzyme is highly conserved, even in organisms that do not have PKC homologs, such as most bacteria. In fact, the appellation of PKCI-1 as an inhibitor of protein kinase $\mathrm{C}$ had been downgraded from heat-stable to heat-labile in 1991 (Fraser \& Walsh, 1991), and there had been no success in replicating this activity, as mentioned above. The resurgence of this annotation is attributed, by Brenner et al., to yeast two-hybrid experiments that expressed fragments of the human HINT cDNA sequence, which matched the defunct PKCI-1 references in protein databases. One of the yeast two-hybrid screens identified PKC- $\beta$ as a binding partner for HINT, but further analysis showed that this was only an artifact of using a HINT fragment, rather than the 
full sequence (Brenner et al., 1999). This 1999 article presented a clear history of why the PKCI-1 annotation had been used and why it is inappropriate to continue using it.

Since this time, the structural literature has been consistent in identifying analogous proteins using the HINT nomenclature (Bardaweel et al., 2010; Ozga et al., 2010; Dolot et al., 2012, 2013; Wang et al., 2012; Maize et al., 2013) until Crystal structure of HINT from Helicobacter pylori (Tarique et al., 2016), which not only reintroduces the abandoned PKCI-1 annotation, but also asserts that PKCI-1 is a distinct family member, rather than just another name for HINT1. It is unclear where the authors got their information, as the papers that they cite in the introduction clearly express either doubt or outright condemnation that HINT inhibits protein kinase C (Lima et al., 1996; Brenner et al., 1999).

\section{References}

Bardaweel, S., Pace, J., Chou, T.-F., Cody, V. \& Wagner, C. R. (2010). J. Mol. Biol. 404, 627-638.

Bieganowski, P., Garrison, P. N., Hodawadekar, S. C., Faye, G., Barnes, L. D. \& Brenner, C. (2002). J. Biol. Chem. 277, 1085210860.

Brenner, C., Bieganowski, P., Pace, H. C. \& Huebner, K. (1999). J. Cell. Physiol. 181, 179-187.
Brenner, C., Garrison, P., Gilmour, J., Peisach, D., Ringe, D., Petsko, G. A. \& Lowenstein, J. M. (1997). Nat. Struct. Mol. Biol. 4, 231238.

Chou, T.-F., Baraniak, J., Kaczmarek, R., Zhou, X., Cheng, J., Ghosh, B. \& Wagner, C. R. (2007). Mol. Pharm. 4, 208-217.

Chou, T.-F. \& Wagner, C. R. (2007). J. Biol. Chem. 282, 4719-4727.

Dolot, R., Ozga, M., Wlodarczyk, A., Krakowiak, A. \& Nawrot, B. (2012). Acta Cryst. F68, 883-888.

Dolot, R., Wlodarczyk, A., Bujacz, G. D. \& Nawrot, B. (2013). Acta Cryst. F69, 783-787.

Fraser, E. D. \& Walsh, M. P. (1991). FEBS Lett. 294, 285-289.

Lima, C. D., Klein, M. G. \& Hendrickson, W. A. (1997). Science, 278, 286-290.

Lima, C. D., Klein, M. G., Weinstein, I. B. \& Hendrickson, W. A. (1996). Proc. Natl Acad. Sci. 93, 5357-5362.

Maize, K. M., Wagner, C. R. \& Finzel, B. C. (2013). FEBS J. 280, 3389-3398.

McDonald, J. R. \& Walsh, M. P. (1985). Biochem. J. 232, 559-567.

Murakami, E., Tolstykh, T., Bao, H., Niu, C., Steuer, H. M. M., Bao, D., Chang, W., Espiritu, C., Bansal, S., Lam, A. M., Otto, M. J., Sofia, M. J. \& Furman, P. A. (2010). J. Biol. Chem. 285, 3433734347.

Ozga, M., Dolot, R., Janicka, M., Kaczmarek, R. \& Krakowiak, A. (2010). J. Biol. Chem. 285, 40809-40818.

Tarique, K. F., Devi, S., Abdul Rehman, S. A. \& Gourinath, S. (2016). Acta Cryst. F72, 42-48.

Wang, J., Fang, P., Schimmel, P. \& Guo, M. (2012). J. Phys. Chem. B, 116, 6798-6805. 
STRUCTURAL BIOLOGY COMMUNICATIONS

ISSN 2053-230X

Received 29 January 2016

Accepted 10 March 2016

Edited by W. N. Hunter, University of Dundee, Scotland

Keywords: HINT; PKCI

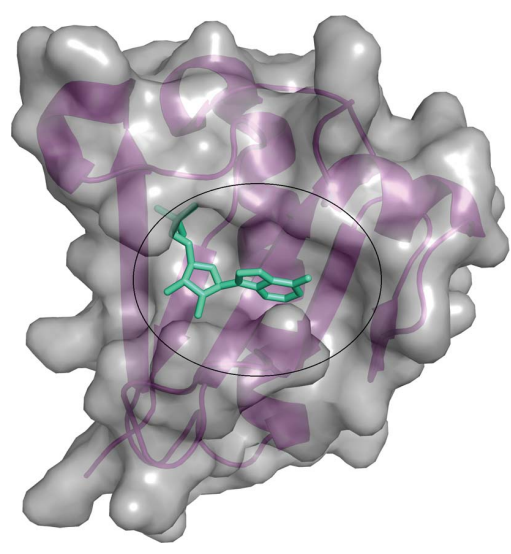

C 2016 International Union of Crystallography

\section{Response to Errors in Crystal structure of HINT from Helicobacter pylori}

\author{
K. F. Tarique, S. Devi, S. A. Abdul Rehman and S. Gourinath* \\ School of Life Science, Jawaharlal Nehru University, New Delhi, Delhi 110 067, India. *Correspondence e-mail: \\ sgourinath@mail.jnu.ac.in
}

A response is published to a Letter to the Editor by Maize [(2016), Acta Cryst. F72, 336-337].

We appreciate and thank Ms Maize for showing interest in our article (Tarique et al., 2016), for writing such a comprehensive critique in her Letter to the Editor (Maize, 2016), and also for raising this issue in the general interest of the scientific community.

Looking at the previous literature for HINT proteins, the nature of this protein has been the topic of much debate as no direct biological function of the protein was known. There are two different views on this protein. Previously, one group of researchers was of the opinion that HINT and PKCI are very similar but that the latter binds and inhibits protein kinase $\mathrm{C}$, while the former does not. Gradually over a period of time the opinion was formed that they are the same protein and the notion that it binds and interacts with protein kinase $\mathrm{C}$ is unproven. However, in the literature the nomenclature for this family of proteins has been found to be repeatedly interchanged. The confusion arises from the annotation of this protein in the respective genomic database of the organism where it is still named as protein kinase $\mathrm{C}$ interacting protein, which should also have been annotated as HINT.

The confusion arises again from the various PDB entries where the same protein was named as PKCI in one entry and HINT in another. Even the latest HINT entry from Entamoeba histolytica has again used the term PKCI and described it as a separate branch of HINT. Even the link maintained by NCBI classifies the HINT subgroup and PKCI related protein as a separate branch of the HIT family (http:// www.ncbi.nlm.nih.gov/Structure/cdd/cddsrv.cgi?uid=238607). Interestingly when we first cloned HINT from Helicobacter pylori it was annotated as PKCI in the NCBI gene bank (http://www.ncbi.nlm.nih.gov/gene/898890). Even the kegg database classifies HINT from H. pylori as PKCI (http://www.genome.jp/dbget-bin/www_bget?hpy:HP0404). The ensemble bacterial genome database again classifies HINT from $H$. pylori as PKCI (http://ensemblgenomes.org/id/AAD07473).

Lorimer et al. (2015) also mentioned that 'EhHIT is similar in amino-acid sequence to proteins that have been given the designation protein kinase $\mathrm{C}$ inhibitor-1 (PKCI-1). This HINT branch has also been identified to contain purine nucleoside- and nucleotidebinding proteins' (3oxk; Lorimer et al., 2015).

As HINT and PKCI are the same then, in our opinion, we prefer not to use the term PKCI at all, even though it is used continually in other places. In the manuscript by Tarique et al. (2016) both old and new references were used and it was inferred that HINT and PKCI are different proteins but with very similar structure. However, we are thankful to Ms Maize who has raised and resolved this issue.

We agree that the substrate for HINT was mistakenly written as phosphoramidite (a typographical error) that should have been written as phosphoramidate.

\section{References}

Lorimer, D. D., Choi, R., Abramov, A., Nakazawa Hewitt, S., Gardberg, A. S., Van Voorhis, W. C., Staker, B. L., Myler, P. J. \& Edwards, T. E. (2015). Acta Cryst. F71, 572-576.

Maize, K. M. (2016). Acta Cryst. F72, 336-337.

Tarique, K. F., Devi, S., Abdul Rehman, S. A. \& Gourinath, S. (2016). Acta Cryst. F72, 42-48. 